\title{
Nuclear Activity and the Conditions of Star-formation at the Galactic Center
}

\author{
Andreas Eckart* \\ I. Physikalisches Institut der Universität zu Köln, Zülpicher Str. 77, D-50937 Köln, Germany; \\ and Max-Planck-Institut für Radioastronomie, Auf dem Hügel 69, D-53121 Bonn, Germany; \\ E-mail: eckarteph1.uni-koeln.de
}

\section{Valencia-S.}

I. Physikalisches Institut der Universität zu Köln, Zülpicher Str. 77, D-50937 Köln, Germany;

\section{B. Shahzamanian, M. Zajacek, L. Moser, M. Parsa}

I. Physikalisches Institut der Universität zu Köln, Zülpicher Str. 77, D-50937 Köln, Germany; and Max-Planck-Institut für Radioastronomie, Auf dem Hügel 69, D-53121 Bonn, Germany;

\section{Subroweit, F. Peissker, N. Sabha, M. Horrobin, C. Straubmeier}

I. Physikalisches Institut der Universität zu Köln, Zülpicher Str. 77, D-50937 Köln, Germany;

\section{A. Borkar, D. Kunneriath, V. Karas}

Astronomical Institute of the Academy of Sciences Prague, Bocni II 1401/1a, CZ-141 31 Praha

4, Czech Republic

\section{Rauch, S. Britzen, A. Zensus}

Max-Planck-Institut für Radioastronomie, Auf dem Hügel 69, D-53121 Bonn, Germany;

\section{García-Marín}

European Space Agency (ESA/STScI), 3700 San Martin Drive, Baltimore, MD 21218, USA

The Galactic Center is the closest galactic nucleus that can be studied with unprecedented angular resolution and sensitivity. We summarize recent basic observational results on Sagittarius A* and the conditions for star formation in the central stellar cluster. We cover results from the radio, infrared, and X-ray domain and include results from simulation as well. From (sub-)mm and near-infrared variability and near-infrared polarization data we find that the SgrA* system (supermassive black hole spin, a potential temporary accretion disk and/or outflow) is well ordered in its geometrical orientation and in its emission process that we assume to reflect the accretion process onto the supermassive black hole (SMBH).

Frontier Research in Astrophysics - II

23-28 May 2016

Mondello (Palermo), Italy

\footnotetext{
*Speaker.
} 


\section{Introduction}

Sagittarius $\mathrm{A}^{*}\left(\operatorname{Sgr} \mathrm{A}^{*}\right)$ at the center of our Galaxy is a highly variable near-infrared (NIR) and $\mathrm{X}$-ray source which is associated with a $4 \times 10^{6} \mathrm{M}_{\odot}$ supermassive central black hole (see discussion in Eckart et al. 2017). This region allows us in an unprecedented way to study at the same time, the nuclear activity associated with accretion onto the supermassive black hole, and the possibility for star formation in its vicinity. Accretion and star formation models must take into account the presence (or influence) of several dusty sources in the central 1 pc region (Meyer et al. 2014, Eckart et al. 2013). The fast motion of one of those infrared excess sources was discovered by Gillessen et al. (2012). They interpret it as a core-less (i.e. no star at its center) gas and dust cloud approaching SgrA* on an elliptical orbit. Eckart et al. (2013ab) present $\mathrm{K}_{s}$-band identifications (from VLT and Keck data) and proper motions of this DSO ${ }^{1}$. Here we present a brief description of the accretion process acting onto $\mathrm{SgrA}^{*}$ from a statistical analysis of emission at different wavelengths. We also explore the geometrical properties of SgrA* accretion flow and of the DSO via infrared polarimetry (Shahzamanian et al. 2015, 2016).

\section{SgrA* Emission Process}

\subsection{Radio/sub-mm monitoring of SgrA*}

Sgr A* undergoes radio millimeter and sub-millimeter variability. Most of this variable flux density is thought to arise from the very central region of the accretion flow onto the supermassive black hole (Fig. 1a; e.g. Moscibrodzka et al. 2009, 2013). In Subroweit et al. (2016), we report on a detailed statistical analysis of the sub-millimeter $345 \mathrm{GHz}$ and radio $100 \mathrm{GHz}$ flux density distribution of Sgr A*. The millimeter radio observations were carried out using the Australia Telescope Compact Array (ATCA) in the years between 2010 and 2014 (Borkar et al. 2016). In the sub-mm wavelength domain, we used data obtained from several Large Apex Bolometer Camera (LABOCA) observing campaigns in the years 2008 to 2014. In addition to this, we used literature data covering the years from 2004 to 2009 , at frequencies very close to $345 \mathrm{GHz}$. The ATCA observations resulted in the detection of six bright flux density excursions of $\mathrm{Sgr} \mathrm{A}^{*}$. During these events the fluxes varied between 0.5 and $1.0 \mathrm{Jy}$. These events lasted for typically 1.5 to 3 hours.

Moser et al. (2016) report serendipitous detections of line emission with the Atacama Large Millimeter/submillimeter Array (ALMA). The observations were carried out within the central parsec around SgrA* at a resolution of up to 0.5 ". From the $100 \mathrm{GHz}$ continuum and line flux densities (example maps are shown in Fig. 2ab), Moser et al. (2016) obtained temperature and density estimates towards different source components. The spectral index $\left(\mathrm{S} \propto v^{\alpha}\right)$ of the synchrotron emitting material in the immediate surroundings of $\mathrm{Sgr} \mathrm{A}^{*}$ is around $\alpha=0.5$ at $100-250 \mathrm{GHz}$ and around $\alpha=0.0$ at $230-340 \mathrm{GHz}$ interval. From earlier interferometric and single dish sub-mm observations and modeling we know that the overall spectrum drops strongly towards the far-infrared at frequencies above about of $350 \mathrm{GHz}$ (Marrone 2006, Marrone et al. 2006a,b, Eckart 2012). Other

\footnotetext{
${ }^{1}$ DSO stands for Dusty S-cluster Object. The source is also called G2 in the literature. The S-cluster is the cluster of high velocity stars surrounding SgrA*; see Eckart\&Genzel (1997). The DSO passed by the central supermassive black hole of the Milky Way SgrA* within 150-160 AU.
} 
Galactic Center regions emitting continuum radiation indicate contributions from Bremsstrahlung (around $\alpha=-0.1$ ) and contributions of cold dust (with flatter/inverted spectral indices).

Subroweit et al. (2016) and Borkar et al. (2016) show that the variable millimeter flux density of SgrA* can be explained via the adiabatically expanding plasmon model. For earlier events Yusef-Zadeh et al. (2006) explained the flaring activity of Sagittarius A* at 43 and $22 \mathrm{GHz}$ as evidence for expanding blob of hot plasma. This phenomenological model was soon developed with much more solid dynamical base and an MHD model for the general picture of plasmoid ejection and associated flare was proposed by Yuan et al. (2009). Most recently, the model was further developed by calculating the detailed radiation of the ejected blob based on dynamics and compared with flare observations of Sgr A* (Li et al. 2017). In Fig. 1 b we show schematically how a sub-mm flare component evolves for three different consecutive times. As a result of the adiabatic expansion, the peak flux decreases and the spectral width of the component increases. These physical parameters derived from the modelling imply that the expanding source components responsible for the flux density variability of $\mathrm{SgrA}^{*}$, are either confined to the immediate vicinity of Sgr A* or they have a bulk motion greater than their expansion velocity. They may be generated in the temporary disk or corona component of SgrA*.

The flux-density variations were not obviously affected by the approach or the flyby of the DSO. This implies that the source must be very compact and does not have a large bow shock.

Subroweit et al. (2016) show that the APEX and ATCA light curves can be described by a shifted power law. The dependency can be written as $\mathrm{p}(\mathrm{x}) \propto(\mathrm{x}-\mathrm{s})^{-\alpha}$ with $\alpha \sim 4$ (APEX: $\alpha=4.0$ \pm 1.7 , ATCA: $\alpha=4.7 \pm 0.8$ ). A similar power law index was found investigating near-infrared (NIR) light curves (Witzel et al. 2012). The value measured in the optically thin NIR parts (Witzel et al. 2012) has been obtained with very good statistics from a large number of lightcurves. The $100 \mathrm{GHz}$ and $345 \mathrm{GHz}$ (sub-)mm values shown in Fig. 3a reflect this NIR value very well. The data points with vertical ranges in Fig. 3a are taken from Subroweit et al. (2016) and Witzel et al. (2012). The horizontal bar reflects the range of synchrotron turn-over frequencies for the case of a synchrotron source with a magnetic field of less than $30 \mathrm{G}$, peak flux densities of less than $2 \mathrm{Jy}$, source sizes of less than 2 Schwarschild radii, and an optically thin spectral index of -0.7 under the assumption that the NIR is still optically thin synchrotron radiation and the X-ray domain stems from Synchrotron Self-Compton (SSC) radiation (Fig.6, Eckart et al. 2012). This region marks where the turnover frequencies of probably most of the bright radio flares are located. These flares then expand adiabatically into the radio, provid the optically thin synchrotron emission in the NIR, and SSC-scatter into the X-ray domain.

The overall findings support our preferred flare emission models in which the adiabatically expanding plasmons dominate the radio - sub-mm short term variability (see Fig. 1ab). When they are most compact they are responsible for the variable optically thin NIR emission and produce variable X-ray emission via the SSC effect.

In the model the flux density variations in all wavelength bands stem from the same source components and statistically they represent a single state red noise process. We find that a typical expansion velocity is of the order of $0.01 \mathrm{c}$ and the dominant portion of variable source components are generated with a peak turn-over frequency in the range of about 300-400 GHz. The contribution of higher frequency emission flares that expand faster or flares that are born with turnover frequencies around $100 \mathrm{GHz}$ is minimal. These stonger flares are stronger and superimposed on the source 

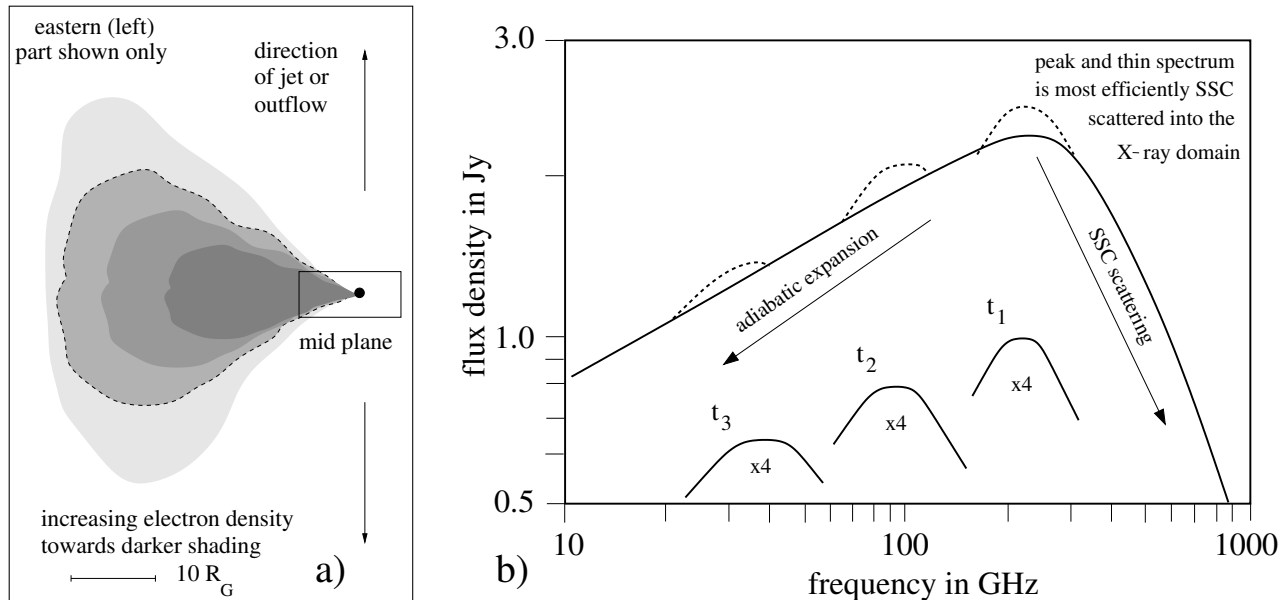

Figure 1: a) Sketch of the density distribution over the azimuthal section across a Sgr A* black hole accretion torus. This depicts the result from relativistic magneto hydrodynamic modeling following Moscibrodzka (2009). The mid-plane is located within the box, to the top and bottom one finds the jet/wind region. b) Depicting schematically the spectral properties of the adiabatic expansion process.

emission fluctuations that consist of the contributions of more extended and (on short times scales) less variable source components.

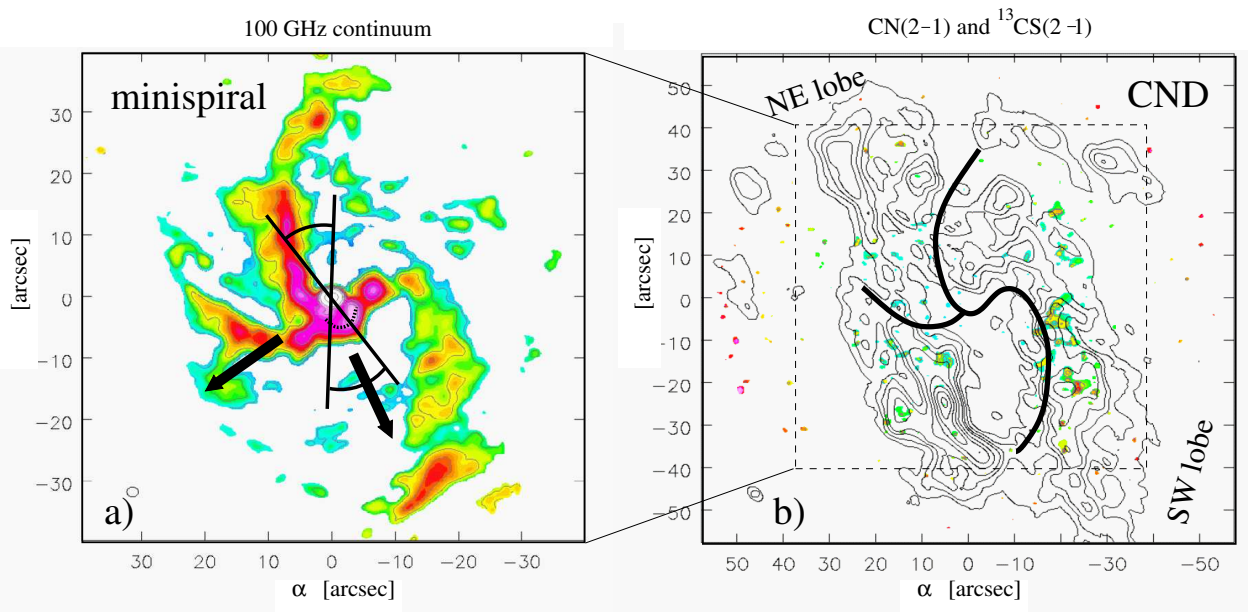

Figure 2: a) ALMA continuum image of the minispiral surounding SgrA* (Moser et al. 2016) and the NIR polarization properties of SgrA*. The conus shows the range over which the polarization angles typically vary. The thick black arrows point into jet directions discussed in the literature (see Shahzamanian et al. 2015). b) The CND in molecular line emission. In contours, as shown by Martin et al. (2012), obtained with the SMA Sub-Millimeter Array at a resolution of (4.0" $\times 2.6 ")$. In color we show the ${ }^{13} \mathrm{CS}(2-1)$ line emission as shown by Moser et al. (2016) with flux densities around $0.2 \mathrm{Jy}$ beam ${ }^{-1} \mathrm{~s}^{-1}$ at a resolution of (1.9" $\left.\times 1.6 "\right)$.

\subsection{Variable radio structure of SgrA*}

In order to determine the nature of some of these (sub-)millimeter flux density excursions we 
performed near-infrared triggered mm-VLBI observations of the radio source Sgr A* at $43 \mathrm{GHz}$ (Rauch et al. 2016). The compact radio and near-infrared source SgrA* associated with the supermassive black hole in the Galactic center was observed in the NIR and mm wavelength range. During a global multiwavelength campaign in May 2012 and based on a NIR flare, we triggered a global Very Long Baseline Array (VLBA) campaign. Here the observations were carried out with the VLBA at $7 \mathrm{~mm}(43 \mathrm{GHz})$ for $6 \mathrm{~h}$ each day. As a result we found that typically the total $43 \mathrm{GHz}$ flux density of Sgr A* shows only minor variations $(\leq 0.06 \mathrm{Jy})$ during its quiescent phases on a daily basis.

We triggered, however, the VLBI observations by a NIR flare observed at the Very Large Telescope (VLT). During this campaign we measured a NIR flare on May 17, 2012, that preceeded a $43 \mathrm{GHz}$ flare of $0.22 \mathrm{Jy}$ by about 4.5 hours. This time delay is consistent with expected values due to adiabatic expansion. Since we were carrying out imaging VLBI observations we could follow the SgrA* source structure during the flare. Close to the peak time of the radio flare, Sgr A* showed a secondary radio component located at about 1.5 mas toward the southeast of the core position. In Fig. $3 \mathrm{~b}$ we show a map at the time of the mm-flare event with the secondary component indicated.

A source component at that distance from the core would be consistent with relativistic bulk motions of the expanding component. Hence, it could be a jet or an outflow component. The event can be explained by a bluk motion at the speed of almost half the speed of light. While variations in the intrinsic source structure have been observed several times (see e.g. Bower et al. 2014), we cannot exclude that radio sub-millimeter scintillations have been involved in the observed effects.
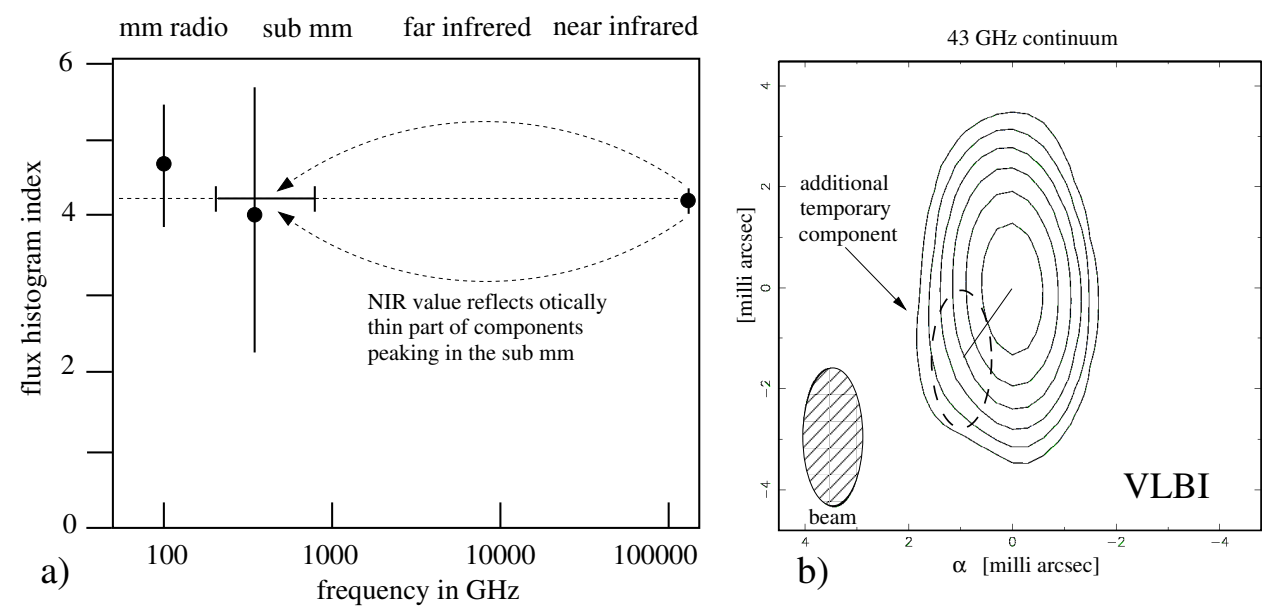

Figure 3: a) Slope of the flare amplitude histograms in different wavelength regimes. b) Right hand circular polarization map of the $43 \mathrm{GHz}$ mm-emission of Sgr A* on May 17, 2012 (8:00-10:00 h UT). The clean beam is shown as a line shaded insert. The contour levels are $1.73 \%, 3.46 \%, 6.93 \%, 13.9 \%, 27.7 \%$, and $55.4 \%$ of the peak flux density of $1.5 \mathrm{Jy} /$ beam (see Rauch et al. 2016 for details).

\subsection{X-ray activity of SgrA*}

With a black hole mass of 4 million solar masses the bolometric luminosity of SgrA* of $L_{b o l} \sim 10^{36} \mathrm{erg} / \mathrm{s}$ is lower than its Eddington luminosity of $L_{E d d}=3 \times 10^{44} \mathrm{erg} / \mathrm{s}$ (Yuan et al. 
2003). Such a low luminosity points at radiatively inefficient accretion flow models (e.g. advection dominated accretion flows - ADAF; Narayan et al. 1998) or models that involve a jet-disk system. SgrA* is not only variable in the radio/sub-mm and infrared domain but also in the X-ray domain. In addition to a 1" diameter quiescent Bremsstrahlung component, there are X-ray flaring events from Sgr A* - first discovered by Baganoff et al. (2001). Typically one finds one bright flare per day (Neilsen et al. 2013) about 10 times the quiescent (2-8 keV) luminosity of SgrA* of about $3.6 \times 10^{33} \mathrm{erg} / \mathrm{s}$ (Baganoff et al. 2003; Nowak et al. 2012). Flares of up to 160 times the quiescent level have been reported (Porquet et al. 2003, 2008; Nowak et al. 2012).

In the NIR we observe optically thin synchrotron radiation emission with a spectral index of about $\alpha=-0.7\left(S_{v} \propto v^{\alpha}\right)$ and a flare amplitude statistics as described by (Witzel et al. 2012). The least demanding requirements to produce the $\mathrm{X}$-ray flux densities appears to hint at a radiation process that involves Comptonization. Here, moderate volume densities $\left(10^{6} \mathrm{~cm}^{-3}\right)$ of low relativistic electrons with $\gamma \sim 10^{3}$ are required (e.g. Eckart et al. 2004 2008, Yusef-Zadeh et al. 2006).

Mossoux et al. (2016) observed a total of seven NIR flares, three of which have XMM-Newton detected X-ray counterparts. Since the flaring rate during this campaign was fully consistent with that of the 2012 Chandra campaign one must conclude that no significantly increased activity was detected during/close to the pericenter passage of the DSO.

Table 1: Conditions for star-formation in the central parsec

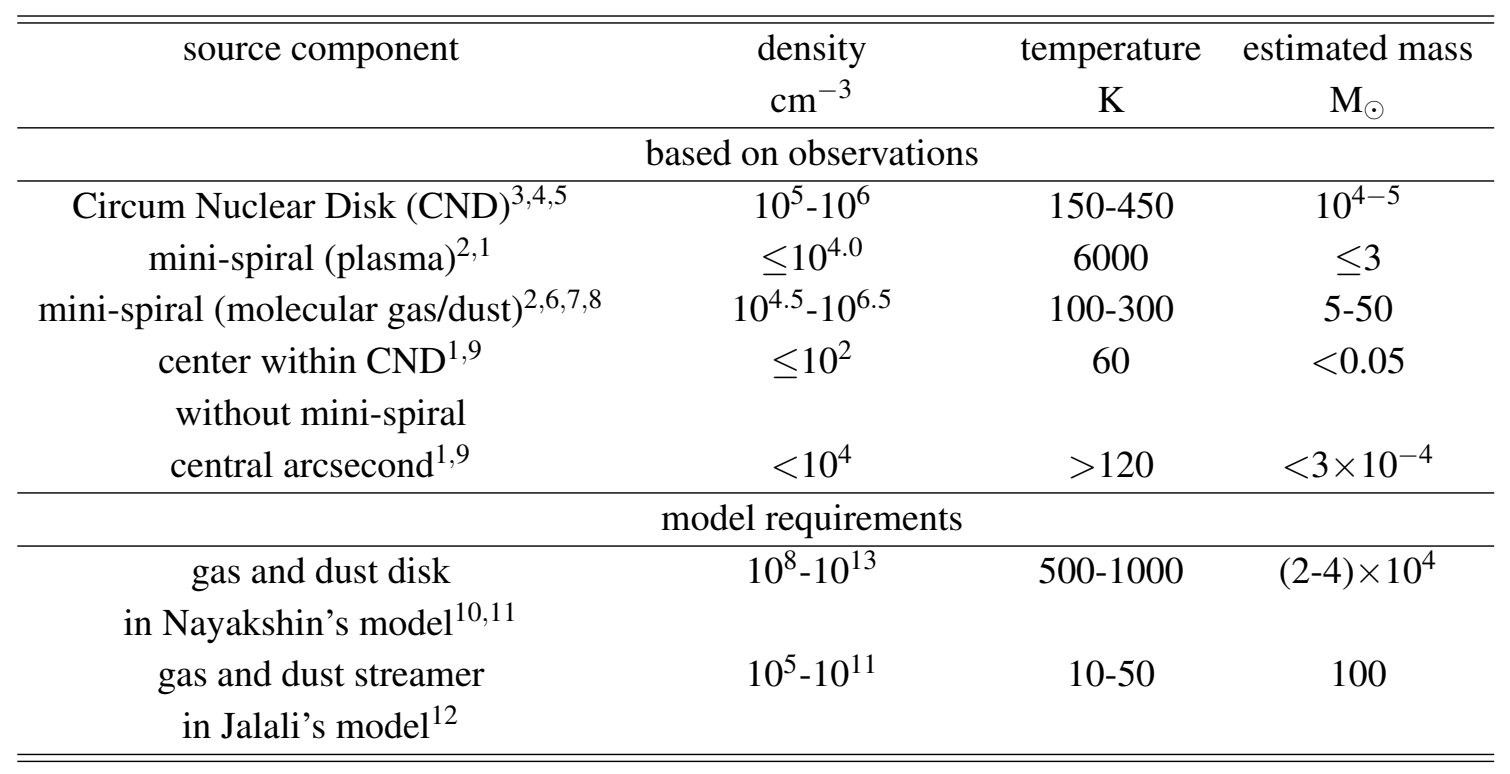

Literature key: 1) Shcherbakov et al. 2010; 2) Moser et al. 2016; 3) Wright et al. 2001; 4) Christopher et al. 2005; (with clumps exceeding densities of $10^{7-8} \mathrm{~cm}^{-3}$ and temperatures of $50 \mathrm{~K}$ or less); 5) Mills et al. 2013; 6) Kunneriath et al. 2012; 7) Jackson et al. 1993; 8) Latvakoski et al. 1999; 9) Rozanska et al. 2014; 10) Nayakshin, Cuadra \& Springel 2007 (with critical density for star formation above $10^{13} \mathrm{~cm}^{-3}$ ); 11) Nayakshin \& Cuadra 2005; 12) Jalali et al. 2014 (with critical density for star formation above $10^{11} \mathrm{~cm}^{-3}$ ). 


\subsection{Infrared Polarimetry of SgrA*}

Polarized radiation gives information on the emission process and the geometry of the emitting source components. In the NIR at a wavelength of $2.2 \mu \mathrm{m}, \mathrm{SgrA} *$ is strongly linearly polarized. Observations have been carried out using the adaptive optics instrument NACO at the VLT UT4 in the infrared $\mathrm{K}_{s}$-band $(2.00 \mu \mathrm{m}-2.36 \mu \mathrm{m})$ from 2004 to 2012 . Several polarized flares were observed during these years, allowing us to study the statistical properties of linearly polarized NIR light from Sgr A*. In Fig. 2a we show how the source is embedded within the mini-spiral (shown in radio continuum emission) and depict the NIR polarization properties. At flux density excursions above $5 \mathrm{mJy}$ (Shahzamanian et al. 2015) the number density histogram for polarized flare fluxes has an exponent $d N / d S \sim 4$, close to the slope of the single state power-law distribution that describes the total power flare flux distribution (Witzel et al. 2012). The polarization degrees are typically around $20 \%$ at a preferred polarization angle of $13^{\circ} \pm 15^{\circ}$. These observational facts imply that the geometry and energetics of the accretion process within the SgrA* system (black hole, wind or jet, and temporary disk or mid-plane as found in simulations; see Fig - see Fig. 1a) are rather stable.

\section{The Dusty S-cluster Object}

In Peissker et al. (2016) we summarize our monitoring of SgrA* and the Dusty S-Cluster Object (DSO) in the near-infrared using VLT SINFONI in the years between 2006 and 2015. The faint DSO was found in 2011 on its way towards the supermassive black hole at the center of our Milky Way. The object was primarily tracked in the thermal NIR L'-band and in its Br $\gamma$-line emission in the NIR K-band. The near-infrared Ks-band continuum emission of the DSO was first reported by Eckart et al. (2013) using NACO at the ESO VLT. It was then also confirmed using the NIRC system at the Keck telescope (Eckart et al. 2014) and SINFONI at the VLT (Eckart et al. 2015). The clear transit between red- and blue-shifted line emission (Valencia et al. 2015), and the fact the the DSO remained a very compact continuum and recombination line emission source (Valencia et al. 2015, Witzel et al. 2014) clearly shows that it did not disintegrate as previously expected (e.g. Gillessen et al. 2012, Pfuhl et al. 2015, Schartmann et al. 2015). Using SINFONI data with high image quality Peissker et al. (2016) now show that the DSO is found as a compact Ks-band line and continuum emitter before and after periapse.

In Shahzamanian et al. (2016) we investigate the near-infrared continuum of the DSO in more detail. For the first time, Shahzamanian et al. (2016) use the near-infrared polarimetric imaging data to determine the polarization of this source. From the data between 2008 and 2012 Shahzamanian et al. (2016) conclude from a robust significance analysis that the DSO is an intrinsically polarized source $(>20 \%)$. It is indicated that the polarization angle varied while it travelled towards the periapse on its orbit around SgrA*. Also, based on the infrared excess of $K_{s}-L>3$, the authors conclude that the DSO might be a dust-enshrouded young star with a compact bow shock forming while it approaches the supermassive black hole (see Fig. 4a). The high polarization degree suggests that the scattering material is arranged in a non-spherical geometry (see e.g. Zajacek, Karas \& Eckart 2014, Zajacek et al. 2016). Shahzamanian et al. (2016) model the DSO as a combination of a bow shock and a bipolar wind of the central star. This model is consistent with the DSO's total 
flux density and the polarization degree. The model naturally explains also the varying polarization angle as being due to intrinsic changes of the source structure, i.e. orientation of the dusty source with respect to the orbit (see Fig. 4b). In addition, an interaction of the DSO with the ambient medium may also influence the polarization angle.

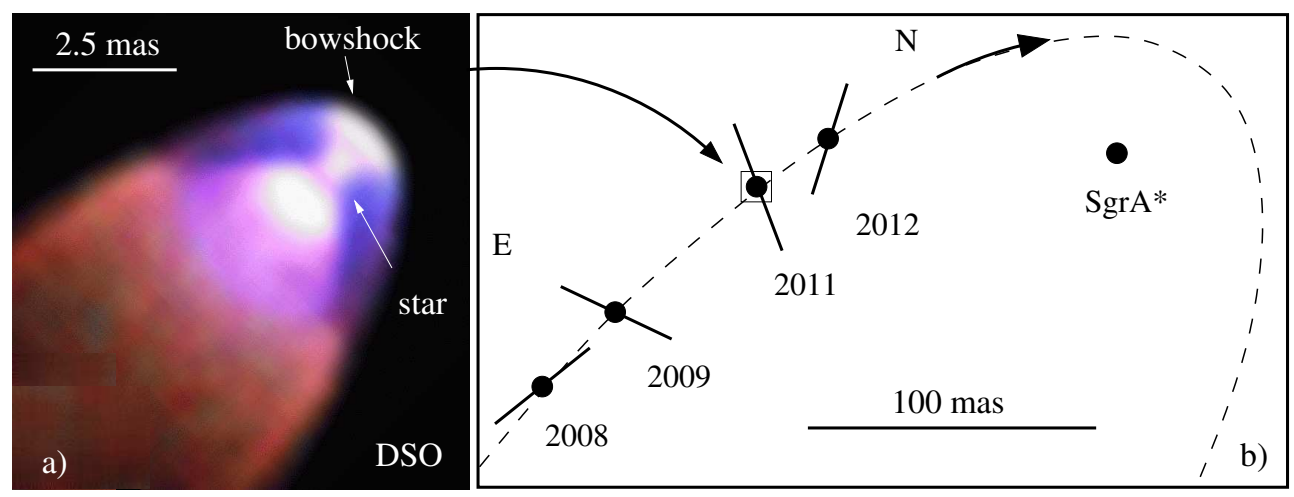

Figure 4: a) An RGB image of the source model of the DSO. While the DSO passes through the medium close to SgrA* it develops a compact bow-shock structure (Shahzamanian et al. 2016 and Zajacek, Karas \& Eckart 2014, Zajacek et al. 2016). b) Polarization properties of the DSO passing by the SgrA* black hole. The polarization degrees are of the order of $30 \%$, the polarization angle varies as shown in the figure (Shahzamanian et al. 2016).

\section{Conditions of Star Formation}

Luminous He-stars in the central few arcseconds (Krabbe et al. 1991 Ghez et al. 2003), the indications for young stars based on colors (Buchholz, Schödel, Eckart 2009), as well as the presence of several dusty objects like the DSO (Meyer et al. 2014, Eckart et al. 2013) suggest that star formation is permanently, or at least episodically, taking place in the central parsec - in the immediate vicinity of the supermassive black hole SgrA*. In Table 1 we list the gas densities and temperatures, as well as the integrated masses for different regions in the central parsec. This table shows that only the CND (Fig. 2b) harbor dense gas entities that may give rise to star formation if a suitable trigger is applied, i.e. additional compression that brings the density above the Jeans density to initiate the collapse to a star. However, for the central few arseconds other mechanisms have to be invoked. It is often assumed that the strong gravitational forces close to SgrA* disrupt dense clouds and prevent classical star formation. However, young stars are observed all over the entire nuclear star cluster. While the massive young He-stars, most of which are located in a disk (Levin \& Beloborodov 2003, Bartko et al. 2008), have most likely been generated in a massive gaseous disk (Nayakshin, Cuadra \& Springel 2007, Nayakshin \& Cuadra 2005), the lower mass stars may have been formed in a different way. Jalali et al. (2014) have shown that molecular clumps of typically $100 \mathrm{M}_{\odot}$ at a radius of less than 0.2 parsec may be subject to black-hole supported star formation due to the orbital compression the clumps experience while they go through periapse, if their temperature is similar to that found in CND clumps. Through dissipative cloud-cloud collisions in the CND the clumps lose angular momentum and can get to pericenter passages close to 
the supermassive black hole. In Table 1 we also list the densities, temperatures and masses covered by the corresponding model calculations. For the Nayakshin case the temperature is higher for a more massive star forming entity, resulting also in more massive stars. The Jalali-mechanism can result in low and intermediate mass star formation that may very well explain the presence of DSO like objects, i.e. compact, dust enshrouded young stars.

\section{Summary}

We have presented results of recent monitoring programs covering the radio to X-ray regime for the Galactic Center as the closest galactic nucleus available. The radio/sub-mm monitoring indicates that the bright 0.1 Jy to a few Jy flare emission of SgrA* can be explained by a simple adiabatic expansion model, in which flares are born within turnover frequencies in the $300-400 \mathrm{GHz}$ range. To first order they are of similar nature, and are consistent with the spectral index and amplitude index information that can be derived from near-infrared and (sub)mm observations. The results from triggered VLBA observations indicate that some of the variable radio components may indeed be traveling outwards as part of a jet or wind. The ordered geometry required for such a scenario is supported by the results from NIR polarization measurements. In general, the flare emission from the radio, near-infrared to X-ray emission may be linked through a synchrotronself-Compton mechanism. Simulations and observations indicate that ongoing star formation at the Galactic Center can be sustained by black-hole supported star formation, either via the formation of a massive star forming disk or by triggering the compression of dense molecular clumps from the CND during periapse on in-falling orbits. The Dusty S-cluster Object may be an example of a young stellar low-mass system.

\section{Acknowledgements}

We received funding from the European Union Seventh Framework Program (FP7/2013-2017) under grant agreement no 312789 - Strong gravity: Probing Strong Gravity by Black Holes Across the Range of Masses. This work was supported in part by the Deutsche Forschungsgemeinschaft (DFG) via the Cologne Bonn Graduate School (BCGS), the Max Planck Society through the International Max Planck Research School (IMPRS) for Astronomy and Astrophysics, as well as special funds through the University of Cologne and SFB 956 âĂŞ Conditions and Impact of Star Formation. M. Zajacek, M. Parsa and B. Shahzamanian are members of the IMPRS. Part of this work was supported by fruitful discussions with members of the European Union funded COST Action MP0905: Black Holes in a Violent Universe and the Czech Science Foundation - DFG collaboration (No. 13-00070J).

\section{References}

[1] Baganoff, F. K., Bautz, M. W., Brandt, W. N., et al. 2001, Nature, 413, 45

[2] Baganoff, F. K., Maeda, Y., Morris, M., et al. 2003, ApJ, 591, 891

[3] Borkar, A.; Eckart, A.; Straubmeier, C.; Kunneriath, D.; et al., 2016, MNRAS 458, 2336

[4] Bartko, H.; Eisenhauer, F.; Fritz, T.; Genzel, R.; et al., 2008, JPhCS 131a2010B 
[5] Bower, G.C.; Markoff, S.; Brunthaler, A.; Law, C.; et al., 2014, ApJ 790, 1

[6] Buchholz, R. M.; Schödel, R.; Eckart, A., 2009, A\&A 499, 483

[7] Christopher, M.H.; Scoville, N.Z.; Stolovy, S.R.; Yun, Min S., 2005, ApJ 622, 346

[8] Eckart, A.; Hüttemann, A.; Kiefer, C.; Britzen, S.; Zajacek, M.; Lämmerzahl, C.; Stöckler, M.; Valencia-S, M.; Karas, V.; Garcia-Marin, M. 2017, Foundation of Physics 47, 553

[9] Eckart, A.; Britzen, S.; Valencia-S., M.; Straubmeier, C.; Zensus, J. A.; Karas, V.; Kunneriath, D.; Alberdi, A.; Sabha, N.; Schödel, R.; Pützfeld, D. The Galactic Center Black Hole Laboratory, 2015arXiv150102171E, 2015 in "Equations of Motion in Relativistic Gravity", D. Puetzfeld et. al. (eds.), Fundamental theories of Physics, 179, pages 759-781, Springer 2015

[10] Eckart, A.; Horrobin, M.; Britzen, S.; Zamaninasab, M.; et al.; Proceedings of the International Astronomical Union, Volume 303, pp. 269-273, 05/2014

[11] Eckart, A., Muzic, K.; Yazici, S.; Sabha, N.; et al., 2013, A\&A 551, 18

[12] Eckart, A.; Garcia-Marin, M.; Vogel, S. N.; et al., 2012, A\&A 537, 52

[13] Eckart, A., Schödel, R.; Garcia-Marin, M.; Witzel, G.; et al. 2008, A\&A 492, 337

[14] Eckart, A.; Baganoff, F. K.; Morris, M.; et al., 2004, A\&A 427, 1

[15] Ghez, A. M.; Duchene, G.; Matthews, K.; et al., 2003, ApJ 586, L127

[16] Gillessen, S., Genzel, R.; Fritz, T. K.; Quataert, E.; et al. 2012, Nature 481, 51

[17] Jalali, B.; Pelupessy, F. I.; Eckart, A.; Portegies Zwart, S.; et al., 2014, MNRAS 444, 1205

[18] Jackson, J. M.; Geis, N.; Genzel, R.; Harris, A. I.; et al., 1993, ApJ 402, 173

[19] Krabbe, A.; Genzel, R.; Drapatz, S.; Rotaciuc, V., 1991, ApJ 382, L19

[20] Kunneriath, D.; Eckart, A.; Vogel, S. N.; Teuben, P.; et al., 2012, A\&A 538, 127

[21] Latvakoski, H. M.; Stacey, G. J.; Gull, G. E.; Hayward, T. L., 1999, ApJ 511, 761

[22] Levin, Y.; Beloborodov, A.M., 2003, ApJ 590, L33

[23] Li, Ya-Ping; Yuan, Feng; Wang, Q. Daniel; 2017, MNRAS 468, 2552

[24] Martin, S., Martin-Pintado, J., Montero-Castano, M., Ho, P. T. P., \& Blundell, R., 2012, A\&A 539, 29

[25] Mills, E.A.C.; Güsten, R.; Requena-Torres, M.A.; Morris, M.R., 2013, ApJ 779, 47

[26] Narayan, R., Mahadevan, R., Grindlay, J. E., et al.; 1998, ApJ, 492, 554

[27] Neilsen, J., Nowak, M. A., Gammie, C., et al. 2013, ApJ, 774, 42

[28] Nowak, M. A., Neilsen, J., Markoff, S. B., et al. 2012, ApJ, 759, 95

[29] Marrone, D.P. 2006, Ph.D. Thesis, Harvard Univ.

[30] Marrone, D.P., Moran, J.M., Zhao, J.-H., \& Rao, R. 2006a, ApJ, 640, 308

[31] Marrone, D.P., Moran, J.M., Zhao, J.-H., \& Rao, R. 2006b, JPhCS, 54, 354

[32] Moser, L.; Sanchez-Monge, A.; Eckart, A.; Requena-Torres, M.A.; Garcia-Marin, M.; et al. 2016arXiv160300801M

[33] Moscibrodzka, M.; Falcke, H., 2013, A\&A 559, L3 
[34] Moscibrodzka, M.; Gammie, C.F.; et al., 2009, ApJ 706, 497

[35] Mossoux, E.; Grosso, N.; Bushouse, H.; Eckart, A.; Yusef-Zadeh, F.; et al., 2016, A\&A 589, 116

[36] Nayakshin, S.; Cuadra, J., 2005, A\&A 437, 437

[37] Nayakshin, S.; Cuadra, J.; Springel, V., 2007, MNRAS 379, 21

[38] Peissker, F.; Valencia-S., M.; Eckart, A.; Parsa, M.; Zajacek, M.; \& Shahzamanian, B.; in prep. 2016

[39] Pfuhl, O.; Gillessen, S.; Eisenhauer, F.; Genzel, R., 2015, ApJ 798, 111

[40] Porquet, D., Predehl, P., Aschenbach, B., et al. 2003, A\&A, 407, L17

[41] Porquet, D., Grosso, N., Predehl, P., et al. 2008, A\&A, 488, 549

[42] Rauch, C.; Ros, E.; Krichbaum, T. P.; Eckart, A.; Zensus, J. A.; Shahzamanian, B.; Muzic, K. 2016, A\&A 587, 37

[43] Rozanska, A.; Czerny, B.; Kunneriath, D.; et al., 2014, MNRAS 445, 4385

[44] Schartmann, M.; Ballone, A.; Burkert, A.; Gillessen, S.; et al.i, 2015, ApJ 811, 155

[45] Shahzamanian, B., Eckart, A., Zajacek, M., Valencia-S., M. , et al., accepted by A\&A, 2016

[46] Shahzamanian, B.; Eckart, A.; Valencia-S., M.; Witzel, G.; et al. 2015, A\&A 576, 20

[47] Shcherbakov, R., Baganoff, F., 2010, ApJ 716, 504

[48] Subroweit, M., Garcia-Marin, M., Eckart, A., Borkar, A., Valencia-S., M., Witzel, G., Shahzamanian, B. , and Straubmeier, C., A\&A, submitted

[49] Valencia-S., Eckart, A., et al. 2015, ApJ 800, 125

[50] Witzel, G., Eckart, A.; Bremer, M.; Zamaninasab, M.; et al., 2012, ApJS 20318

[51] Witzel, G.; Ghez, A. M.; Morris, M. R.; et al., 2014, ApJ 796, L8

[52] Wright, M.C.H.; Coil, A.L.; McGary, R.S., et al., 2001, ApJ 551, 254

[53] Yuan, F., Quataert, E., \& Narayan, R. 2003, ApJ, 598, 301

[54] Yuan, Feng; Lin, Jun; Wu, Kinwah; Ho, Luis C.; 2009, MNRAS 395, 2183

[55] Yusef-Zadeh, F.; Bushouse, H.; Dowell, C. D.; et al., 2006, ApJ 644, 198

[56] Yusef-Zadeh, F.; Roberts, D.; Wardle, M.; Heinke, C. O.; Bower, G. C., 2006, ApJ 650, 189

[57] Zajacek, M.; Karas, V.; Eckart, A., 2014, A\&A 565, 17

[58] Zajacek, M.; Eckart, A.; Karas, V.; Kunneriath, D.; Shahzamanian, B.; Sabha, N.; Muzic, K.; Valencia-S., M.; 2016, MNRAS 455, 1257 